\title{
A New Online Updating Framework for Constructing Monotonicity-Preserving Fuzzy Inference Systems
}

\author{
${ }^{* 1}$ Kai Meng Tay, ${ }^{1}$ Tze Ling Jee, ${ }^{1}$ Lie Meng Pang, ${ }^{2}$ Chee Peng Lim \\ ${ }^{1}$ Faculty of Engineering, \\ Universiti Malaysia Sarawak, \\ Sarawak, Malaysia \\ ${ }^{2}$ Centre for Intelligent Systems Research, \\ kmtay@feng.unimas.my \\ Deakin University, \\ Australia.
}

\begin{abstract}
In this paper, a new online updating framework for constructing monotonicity-preserving Fuzzy Inference Systems (FISs) is proposed. The framework encompasses an optimization-based Similarity Reasoning (SR) scheme and a new monotone fuzzy rule relabeling technique. A complete and monotonically-ordered fuzzy rule base is necessary to maintain the monotonicity property of an FIS model. The proposed framework attempts to allow a monotonicity-preserving FIS model to be constructed when the fuzzy rules are incomplete and not monotonically-ordered. An online feature is introduced to allow the FIS model to be updated from time to time. We further investigate three useful measures, i.e., the belief, plausibility, and evidential mass measures, which are inspired from the DempsterShafer theory of evidence, to analyze the proposed framework and to give an insight for the inferred outcomes from the FIS model.
\end{abstract}

Keywords-Fuzzy inference system; Monotonicity; Online updating; Fuzzy rule relabeling; Optimization-based similarity reasoning; Belief; Plausibility; Evidential mass

\section{INTRODUCTION}

To maintain the monotonicity property of a Fuzzy Inference System (FIS) model, a monotonically ordered and complete fuzzy rule base is necessary [1-6]. However, in many practical applications, it is difficult to ensure a monotonically ordered and complete fuzzy rule base. The focus of this paper is on two of our proposed techniques, i.e., monotone fuzzy rule relabeling [7] and optimization-based Similarity Reasoning (SR) [7-8, 13-14], for constructing a monotonicity-preserving FIS. The former technique, i.e., monotone fuzzy rule relabeling, attempts to relabel a non-monotone (and maybe incomplete) fuzzy rule base gathered from domain experts [7]. It searches for a new fuzzy rule base with three aims, viz., monotone (as the first priority), minimum number of relabeled rules (as the second priority), and minimum loss measure (as the third priority).

The later, i.e., the optimization-based SR scheme [7-12], attempts to maintain a monotonically ordered and complete fuzzy rule base. From the literature, various SR schemes (e.g., analogical reasoning [9], fuzzy rule interpolation [10-11], and qualitative reasoning [12]) are available to allow the conclusion of an observation (in the form of a fuzzy set) to be deduced or predicted, based on a fuzzy rule base (database). Even though these approaches are useful, it is difficult to ensure a monotonically-ordered fuzzy rule base to be obtained [7-8, 1314]. Thus, we argue that the deduced conclusions need to be optimized, before implementing them in practice [7-10]. It is worth mentioning that in our previous investigations, applications of SR to failure mode and effect analysis methodology [9] and education assessment [10] have been investigated.

The first objective of this paper is to propose a new framework i.e., an online (potentially real time) updating framework that encompasses the optimization-based SR scheme and a new monotone fuzzy rule relabeling technique [15]. While many investigations on SR have been reported, the applications of SR are still limited. The proposed framework demonstrates the potential of SR and a new monotone fuzzy rule relabeling technique in various practical application domains. The proposed framework also provides a solution to modeling an FIS model when the fuzzy rules are incomplete and not monotonically ordered. It allows new fuzzy rules to be updated from time to time. In our previous works [7-8, 13-14], it is assumed that the fuzzy rules are static, and it is unclear how to update the FIS model when new information i.e., new fuzzy rule(s), is provided. The proposed framework is an extension of our previous works, in which an online updating feature is introduced.

While the proposed framework is able to deduce a monotone outcome, it is not explicit whether the deduced outcome is valid. In order to give an insight for the inferred outcomes, three new measures which are inspired from the Dempster-Shafer Theory (DST) of evidence [16], i.e., the belief, plausibility, and evidential measures, are further investigated. The belief measure (i.e., bel, where $0 \leq$ bel $\leq$ 1) indicates a measure of evidence (i.e., fuzzy rule(s) from the domain experts) that supports the outcome. The plausibility measure (i.e., $p l$, where $0 \leq p l \leq 1$ ) indicates a measure of evidence (i.e., fuzzy rule(s) from the domain experts) that is against the outcome. There are two sources of evidence (i.e., fuzzy rule(s)) that are against the outcome, i.e., relabeled fuzzy rules and previous fuzzy rules. The evidential mass measure (i.e., $m a$, where $0 \leq m a \leq 1$ ) indicates a measure of indeterminate evidence, i.e., fuzzy rule(s) that are approximated by SR.

This paper is organized as follows. The background of FIS and its monotonicity property, and the related works of SR are presented in section II. In section III, a new fuzzy rule relabeling technique is firstly described. The new online updating framework is explained. In section IV, the belief, plausibility, and evidential mass measures are defined, and their usefulness are demonstrated. Finally concluding remarks are presented in section $\mathrm{V}$. 